\title{
Elevages de bétail dans la région du Poro (Côte d'Ivoire) : Caractérisation et modalités de lutte contre les pathogènes transmis par les tiques
}

\author{
Nawolo YEO ${ }^{1}$, Yahaya KARAMOKO ${ }^{*}$, Dofara $\mathrm{SORO}^{1}$, Zahouli Faustin ZOUH BI ${ }^{1}$ et \\ Seïdina Ibrahima TRAORE ${ }^{2}$ \\ ${ }^{1}$ Université Nangui Abrogoua, UFR-SN, Laboratoire de Biologie et Cytologie Animales, Pôle de Production \\ Animale 02 BP 801 Abidjan 02, Côte d'Ivoire. \\ ${ }^{2}$ LANADA, Laboratoire Régional de Korhogo (LRK), BP 32 Korhogo, Côte d'Ivoire. \\ *Auteur correspondant ; E-mail: y.karamoko@gmail.com
}

\section{RESUME}

Une enquête a été menée dans les élevages de bétail des départements de la Région du Poro en Côte d'Ivoire. Le but était de caractériser les élevages et rechercher les moyens de lutte contre les infections transmises par les tiques. Un questionnaire a été administré à 120 éleveurs. Les enquêtes ont révélé que, la conduite mixte est observée dans tous les départements à des fréquences variant de $56,67 \%$ à $80 \%$. En outre, les éleveurs confondent les maladies provoquées par les tiques à d'autres maladies du bétail. L'Oxytétracycline $(41,39 \%)$ et l'Amitraz $(82,88 \%)$ étaient les médicaments vétérinaires les plus utilisés dans la Région du Poro. Leur forte utilisation par les éleveurs est observée dans les élevages des départements de Sinématiali et Korhogo. Les doses d'antibiotiques et les dilutions des acaricides n'étaient pas appropriées. Les sous-dilutions et les surdosages des médicaments ont été plus observés. Une étude parasitologique dans la Région du Poro nous permettra d'inventorier les parasites transmis par les tiques afin de mieux proposer un plan de lutte efficace. L'identification de ces hémoparasites permettra aux éleveurs de mieux traiter le bétail et réduire les dépenses effectuées.

(C) 2017 International Formulae Group. All rights reserved.

Mots clés : Enquête, fréquence, élevage bétail, Côte d'Ivoire.

\section{Cattle Breeding in the Poro area (Côte d'Ivoire): Characterisation and of tick- borne diseases control modalities}

\begin{abstract}
A survey was carried in livestock farms in the departments of Poro area of Côte d'Ivoire. The aim was to characterize the breeding and to investigate the methods used to control tick-borne infections in cattle breeding. The survey was managed with 120 stock breeders. The survey revealed that, mixed control was observed in all departments at frequencies ranging from $56.67 \%$ to $80 \%$. In addition, stock breeders confuse tick diseases with other diseases. Oxytetracycline (41.39\%) and Amitraz (82.88\%) were most observed in the Poro area. Their high use by breeders was met in the departments of Sinematiali and Korhogo. Antibiotic
\end{abstract}


amounts and acaricides dilution were not suitable. Under-dilutions and drugs overdoses were observed. A parasitologic study in Poro area will enable us to inventory ticks parasites in order to propose a fight plan. The identification of tick blood parasites will make it possible to stock breeders to better treat the cattle and to reduce the expenditure carried out.

(C) 2017 International Formulae Group. All rights reserved.

Keywords: Survey, frequency, Cattle breeding, Côte d'Ivoire.

\section{INTRODUCTION}

La Côte d'Ivoire dépend habituellement de la région sahélo-soudanienne pour son approvisionnement en viande de bétail (Diallo, 2007 ; Azokou et al., 2016). La zone Nord du pays constitue la principale zone d'élevage des ruminants domestiques. Selon Tangui (2004), les 2/3 de l'effectif du cheptel national bovin sont rencontrés dans les Régions du Poro et du Tchologo du District des Savanes. Malgré les efforts consentis par l'Etat pendant plusieurs décennies, la production nationale en viande reste tout de même déficitaire avec $55,4 \%$ pour la viande et les abats et $87,4 \%$ pour les produits laitiers (Yabile, 2011). Cette situation amplifiée par la crise socio-politique de 2002 a favorisé la résurgence de nombreuses maladies parmi lesquelles, celles causées par les tiques: l'anaplasmose, la babésiose, la theileriose et l'ehrlichiose. Les deux premières maladies sont provoquées par les tiques du genre Boophilus. Tandis que, la theileriose et l'ehrlichiose sont respectivement causées par les tiques $\mathrm{du}$ genre Rhipicephalus et Hyalomma puis Amblyomma (Morel, 2000). Les parasites transmis par les tiques chez les ruminants domestiques sont nombreux parmi lesquels Anaplasma bovis, Anaplasma marginale et Anaplasma centrale sont les agents causant l'anaplasmose. Par contre, Babesia bovis et Babesia bigemina causent la babésiose. Theileria parva, Theileria annulata et Theileria mutans causent la theileriose. Quant à l'ehrlichiose, elle est provoquée par Amblyomma variegatum (Morel, 2000; Rebaud, 2006). Ces parasites sanguins provoquent des avortements, les chutes de poids et la baisse de la production laitière (Farougou et al., 2007; Achi et al., 2012).
L'anémie et la mortalité se chiffrent à près de $50 \%$ chez le bétail (Minjauw et McLeod, 2003). Les pertes annuelles dues aux maladies transmises par les tiques sont estimées à 17,33 milliards de Dollars dans le monde et il est estimé que $80 \%$ de la population du bétail mondial sont exposés aux tiques et aux maladies qu'elles transmettent (Djakaridja et al., 2014). Il est important de s'intéresser davantage aux parasites sanguins transmis par les tiques. Le but était de caractériser les élevages et rechercher les moyens de lutte contre les infections transmises par les tiques. Cette enquête permettra d'apprécier les modes de diagnostic et de lutte contre les maladies transmises par les tiques par les éleveurs dans les élevages.

\section{MATERIEL ET METHODES \\ Milieu d'étude}

L'enquête a concerné quatre départements de la région du Poro de Côte d'Ivoire : Sinématiali, Korhogo, M'Bengué et Dikodougou (Figure 1). Le choix de cette région réside dans le fait qu'elle enregistre une très forte densité d'élevage de ruminants domestiques d'une part et une forte présence de tiques signalée dans les fiches de rapport pathologiques des élevages de la zone des savanes, d'autre part. La zone d'étude fait frontière au Mali et au Burkina Faso. Elle est principalement caractérisée par son climat de type tropical soudanien, avec une saison de pluie qui s'étend d'avril à octobre et une saison sèche qui part de novembre à mars et placée sous l'influence de l'harmattan.

\section{Enquête dans les départements visités}

L'enquête a été menée dans les quatre départements de la Région du Poro. Dans 
chacun de ces départements, 30 éleveurs ont été interrogés soit un effectif de 120 éleveurs dans l'ensemble des quatre départements. Le choix des villages et des éleveurs par département a été fait en fonction de la disponibilité des éleveurs. Les données ont été recueillies à l'aide d'un questionnaire élaboré à cet effet. L'enquête a été menée sur les parcs à bétail, sur le pâturage ou les lieux d'habitation des éleveurs grâce à un traducteur. Vingt-quatre (24) villages ont été visités dans le département de Sinématiali, 25 villages à Korhogo, 14 villages à M'Bengué et 10 villages à Dikodougou. Les données obtenues de l'enquête ont porté essentiellement sur le mode de conduite des animaux ainsi que la reconnaissance de la transmission des parasites par les tiques au bétail ont été renseignés. L'enquête a porté également sur le mode d'utilisation des médicaments vétérinaires pour le traitement $\mathrm{du}$ bétail contre les tiques et les parasites qu'elles transmettent.

\section{Traitement et analyses statistiques des données}

Les fréquences des différents paramètres abordés ont été calculées. Les analyses statistiques ont été effectuées à partir $\mathrm{du}$ logiciel $\mathrm{R}$ version 2.12.1. Le test $\mathrm{G}$ qui est l'équivalent du Khi deux $\left(\chi^{2}\right)$ a servi à comparer les fréquences. Les fréquences ont été considérées significatives au seuil de 5\%.

\section{RESULTATS}

\section{Mode de conduite du bétail dans les différentes localités enquêtées}

Dans toutes les localités visitées, les éleveurs interrogés ont recours à la conduite mixte. Les bovins et les ovins sont conduits sur le même pâturage par le même bouvier. Ces pratiques sont plus observées dans les localités de Sinématiali et M'Bengué (80\%), Korhogo (76,67\%) et Dikodougou (56,67\%). Cette pratique d'élevage est significative $(\mathrm{P}<$ 0,05 ) dans les départements visités (Figure 2).

\section{Niveau de connaissance des maladies transmises par les tiques}

Dans les départements enquêtés plus de $70 \%$ des éleveurs interrogés savent que les tiques transmettent des parasites aux animaux. Les fortes proportions sont observées respectivement dans les localités de Dikodougou (96,67\%) et Korhogo (90\%) (Figure 3).

\section{Utilisation des antibiotiques et des acaricides dans les élevages}

Les éleveurs sédentaires, ont la plupart recours aux acaricides $(94,64 \%)$ contre $12,5 \%$ pour les transhumants dans la lutte contre les tiques. Par contre, moins de la moitié des éleveurs sédentaires $(37,5 \%)$ ont recours aux antibiotiques contre $1,56 \%$ pour les transhumants dans la lutte contre les parasites transmis par les tiques. La forte utilisation des antibiotiques a été observée dans la localité de M'Bengué $(69,23 \%)$ contre $5,88 \%$ pour les transhumants dans cette même localité. Par ailleurs, il est à observer une forte utilisation des acaricides chez les éleveurs sédentaires dans toutes les localités (Tableau 1). La différence d'utilisation des antibiotiques et des acaricides chez les éleveurs sédentaires et transhumants s'est révélée significative $(\mathrm{P}<$ $0,05)$ dans les différentes localités.

\section{Inventaire et proportion des molécules utilisées dans la Région du Poro}

L'analyse du Tableau 2 montre que les éleveurs font recours dans le traitement de leurs animaux à trois molécules antiparasitaires (Diminazène diacéturate, Ivermectine et Chlorydrate de chlorure d'Isométamidium) et trois molécules antibiotiques (Oxytétracycline, Tylosine, et Péniciline). Parmi ces molécules, l'Oxytétracycline était la molécule la plus employée par les éleveurs. La fréquence d'utilisation était de 41,39\%. Les proportions d'emploi des antibiotiques par les éleveurs étaient significatives $(\mathrm{p}<0,05)$. Par ailleurs, l'Amitraz 12,5\% EC et l'Alphacyperméthrine étaient les acaricides rencontrés dans la région 
du Poro avec l'Amitraz, la molécule la plus employée par les éleveurs. La fréquence d'emploi de cette molécule était de $82,88 \%$. Les fréquences d'emploi de ces acaricides étaient significatives $(\mathrm{p}<0,05)$.

\section{Dilution des antiparasitaires et antibiotiques dans les différentes localités enquêtées}

Dans la Région du Poro, $85,71 \%$ des éleveurs sédentaires injectent au bétail des doses de médicament faibles dans la lutte contre les hémoparasites. La proportion des éleveurs sédentaires optant pour des doses normales a été de $14,29 \%$. Le fort sous-dosage des médicaments a été observé chez les éleveurs des localités respectives de M'Bengué $(100 \%)$, Korhogo $(83,33 \%)$ et Sinématiali (75\%). Les cas de surdosage ont été absents chez les éleveurs sédentaires. La différence de dilution des médicaments s'est révélée significative $(\mathrm{P}<0,05)$ dans les différentes localités à l'exception de Dikodougou. Par contre, chez les transhumants, le surdosage des médicaments a été observé seulement chez tous les éleveurs transhumants de la localité de M'Bengué (Tableau 3).

\section{Dilution des acaricides dans les différentes localités enquêtées}

Deux formes de dilution des acaricides (Amitraz 12,5\% EC et Alphacyperméthrine) sont observées chez les sédentaires et les transhumants. Il s'agit de la dilution normale et la sur-dilution. La majorité des éleveurs ont recours à la sur-dilution des acaricides. Cette forte pratique de sur-dilution des acaricides est observée chez les transhumants et les sédentaires dans toutes les localités sauf la localité de M'Bengué (Tableau 4). La différence de proportion de dilution des acaricides est significative $(\mathrm{P}<0,05)$ pour les sédentaires dans les localités enquêtées.

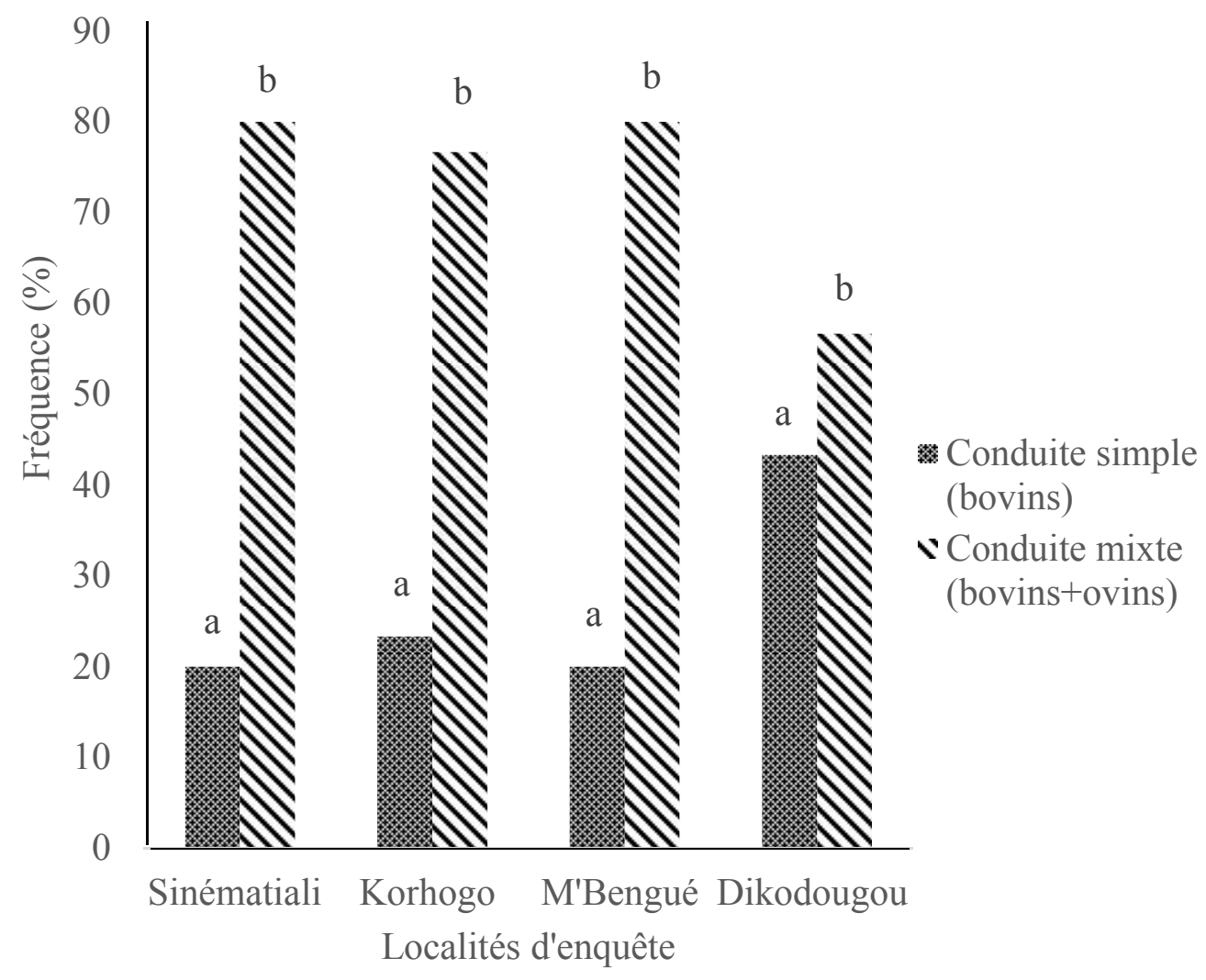

Figure 2: Proportion des formes de conduite du bétail dans les différentes localités enquêtées. 


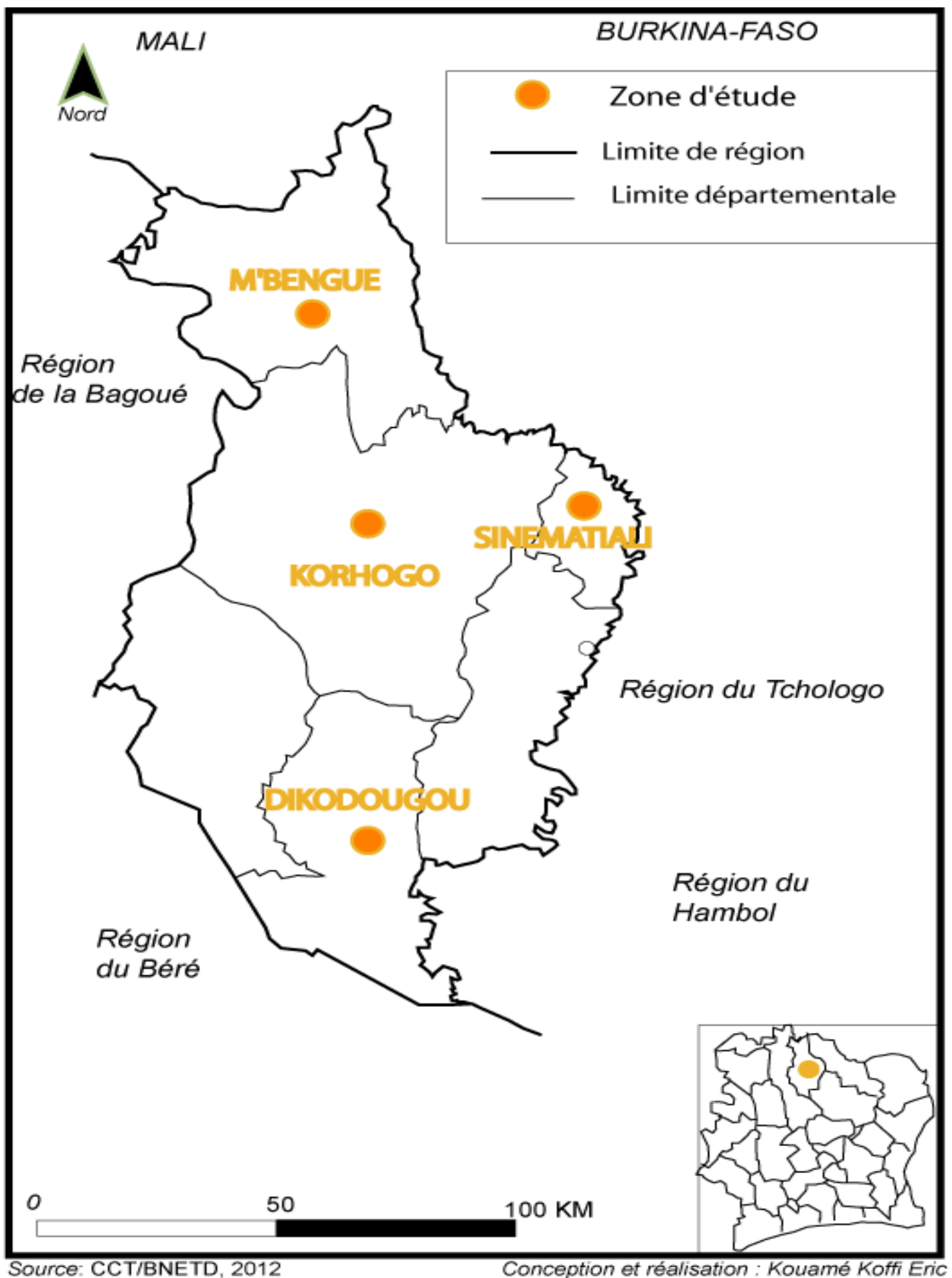

Figure 1 : Carte de la Région du Poro de Côte d'Ivoire situant les départements d'étude. 


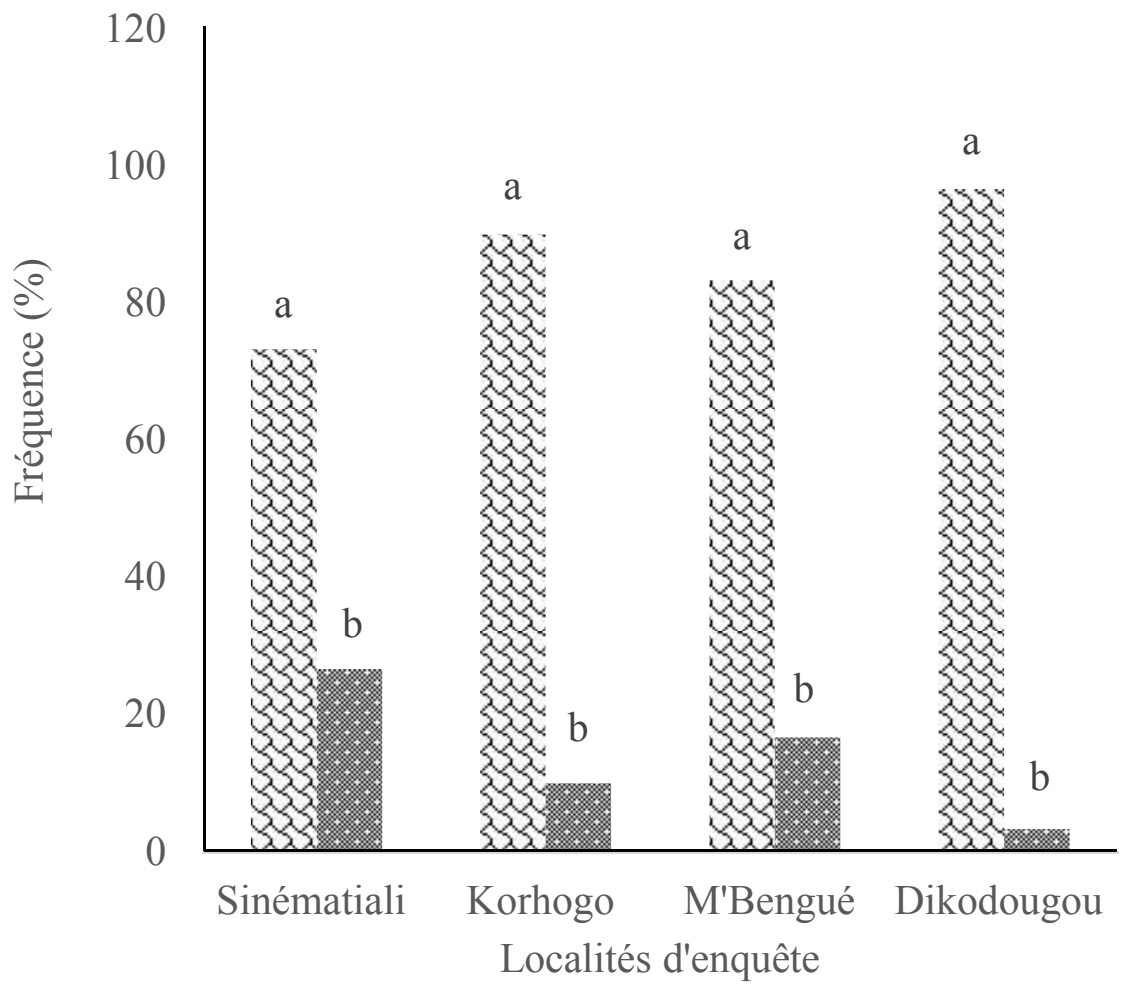

¿Reconnaissance

Reconnaissance

Figure 3: Proportion des éleveurs en fonction de la reconnaissance des hémoparasites transmis par les tiques au bétail dans les localités enquêtées.

Tableau 1: Proportion des éleveurs sédentaires et transhumants utilisant les antibiotiques et les acaricides dans les différentes localités.

\begin{tabular}{|c|c|c|c|c|c|c|}
\hline \multirow{2}{*}{$\begin{array}{l}\text { Localités } \\
\text { d'enquête }\end{array}$} & \multicolumn{2}{|c|}{ Antibiotiques } & \multicolumn{2}{|c|}{ Acaricides } & \multicolumn{2}{|c|}{$\mathbf{P}>\mathbf{F}$} \\
\hline & sédentaire & transhumant & sédentaire & transhumant & Anti. & Acar. \\
\hline Sinématiali & 28,57 & 0 & 100 & 18,75 & - & $\begin{array}{c}< \\
0,001\end{array}$ \\
\hline Korhogo & 26,09 & 0 & 100 & 14,29 & - & $\begin{array}{c}< \\
0,001\end{array}$ \\
\hline M'Bengué & 69,23 & 5,88 & 84,62 & 11,76 & $<0,001$ & $\begin{array}{c}< \\
0,001\end{array}$ \\
\hline Dikodougou & 33,33 & 0 & 83,33 & 8,33 & - & $\begin{array}{c}< \\
0,001\end{array}$ \\
\hline Total & 37,5 & 1,56 & 94,64 & 12,5 & $<0,001$ & $\begin{array}{c}< \\
0,001\end{array}$ \\
\hline
\end{tabular}


Tableau 2 : Inventaire et proportion des molécules utilisés dans la Région du Poro.

\begin{tabular}{llccc}
\hline $\begin{array}{l}\text { Type de } \\
\text { molécule }\end{array}$ & Molécules & $\begin{array}{l}\text { Nombre d'éleveurs } \\
\text { par type de molécule }\end{array}$ & $\begin{array}{l}\text { Nombre } \\
\text { d'éleveurs par } \\
\text { molécule }\end{array}$ & $\begin{array}{c}\text { Proportion } \\
\text { (\%) }\end{array}$ \\
\hline \multirow{2}{*}{ Antiparasitaires } & Diminazène diacéturate & 29 & 5 & $17,24^{\mathrm{a}}$ \\
& Ivermectine & 29 & 3 & $10,34^{\mathrm{a}}$ \\
& Chlorydrate de chlorure & 29 & 5 & $17,24^{\mathrm{a}}$ \\
\hline \multirow{3}{*}{ Antibiotiques } & d'Isométamidium & 29 & 1 & $3,45^{\mathrm{a}}$ \\
& Tylosine & 29 & 12 & $41,39^{\mathrm{b}}$ \\
& Oxytétracycline & 29 & 3 & $10,34^{\mathrm{a}}$ \\
\hline \multirow{2}{*}{ Acaricides } & Péniciline & 111 & 92 & $82,88^{\mathrm{a}}$ \\
& Amitraz 12,5\% EC & 111 & 19 & $17,12^{\mathrm{b}}$ \\
\hline
\end{tabular}

Les valeurs de la même colonne portant la même lettre en exposant ne diffèrent pas significativement au seuil de $0,05 \%$ pour chaque type de molécule.

Tableau 3: Proportion des éleveurs en fonction de la dilution des antibiotiques dans les différentes localités.

\begin{tabular}{|c|c|c|c|c|c|c|c|c|}
\hline \multicolumn{9}{|c|}{ Antibiotique } \\
\hline \multirow{2}{*}{$\begin{array}{l}\text { Localités } \\
\text { d'enquête }\end{array}$} & \multicolumn{3}{|c|}{ Sédentaires } & \multicolumn{3}{|c|}{ transhumants } & \multicolumn{2}{|c|}{$\mathbf{P}>\mathbf{F}$} \\
\hline & $\begin{array}{c}\text { dose } \\
\text { normale }\end{array}$ & $\begin{array}{c}\text { sous } \\
\text { dosage }\end{array}$ & $\begin{array}{c}\text { sur } \\
\text { dosage }\end{array}$ & $\begin{array}{c}\text { dose } \\
\text { normale }\end{array}$ & $\begin{array}{c}\text { sous } \\
\text { dosage }\end{array}$ & $\begin{array}{c}\text { sur } \\
\text { dosage }\end{array}$ & Sed. & Tran. \\
\hline Sinématiali & 25 & 75 & - & - & - & - & $<0,001$ & - \\
\hline Korhogo & 16,67 & 83,33 & - & - & - & - & $<0,001$ & - \\
\hline M'Bengué & - & 100 & - & - & - & 100 & - & - \\
\hline Dikodougou & 50 & 50 & - & - & - & - & $<1$ & - \\
\hline Total & 14,29 & 85,71 & - & - & - & 100 & $<0,001$ & - \\
\hline
\end{tabular}

Tableau 4: Proportion des éleveurs en fonction de la dilution des acaricides dans les différentes localités.

\begin{tabular}{lccccccccc}
\hline \multicolumn{10}{c}{ Acaricides } \\
\hline \multirow{2}{*}{$\begin{array}{c}\text { Localités } \\
\text { d'enquête }\end{array}$} & $\begin{array}{c}\text { Sédentaires } \\
\text { dilution } \\
\text { normale }\end{array}$ & $\begin{array}{c}\text { sous } \\
\text { dilution }\end{array}$ & $\begin{array}{c}\text { sur } \\
\text { dilution }\end{array}$ & $\begin{array}{c}\text { dilution } \\
\text { normale }\end{array}$ & $\begin{array}{c}\text { sous } \\
\text { dilution }\end{array}$ & $\begin{array}{c}\text { sur } \\
\text { dilution }\end{array}$ & Sed. & Tran. \\
\hline Sinématiali & 7,14 & - & 92,86 & - & - & 100 & $<0,001$ & - \\
Korhogo & 8,7 & - & 91,3 & - & - & 100 & $<0,001$ & - \\
M'Bengué & 45,45 & - & 54,55 & 50 & - & 50 & $<0,363$ & $<1$ \\
Dikodougou & 20 & - & 80 & - & - & 100 & $<0,001$ & - \\
\multicolumn{1}{c}{ Total } & 16,98 & - & 83,02 & 12,5 & - & 87,5 & $<0,001$ & $<0,001$ \\
\hline
\end{tabular}




\section{DISCUSSION}

Cette enquête menée dans la Région du Poro a révélé que plus de $70 \%$ des éleveurs interrogés ont connaissance des maladies transmises par les tiques. En effet, les symptômes décrits par les éleveurs (poils piqués, les larmoiements, la cachexie) sont observés aussi dans les infections causées par la trypanosomose. Seul, le diagnostic de laboratoire permet de confirmer la présence des maladies transmises par les tiques. Azokou et al. (2016) ont obtenu des résultats similaires dans une étude conduite chez les éleveurs bovins du Nord de la Côte d'Ivoire.

Les éleveurs sédentaires, ont la plupart recours aux acaricides $(94,64 \%)$ contre $12,5 \%$ pour les transhumants dans la lutte contre les tiques. Cette forte utilisation des acaricides chez les sédentaires pourrait s'expliquer par la persistance des acariens sur les animaux. Cette forte charge des tiques sur les animaux est favorisée par la colonisation des aires de pâturage. Par contre, le changement des parcours chez les transhumants pourrait être à l'origine de la diminution de la charge des acariens sur le bétail. Ces résultats sont contraires à ceux obtenus par Lancelot et al. (2011). Selon ces auteurs, les conditions d'élevage et la mobilité animale contribuent à la diffusion des maladies à transmission directe comme la péripneumonie contagieuse bovine ou la peste des petits ruminants. De plus, l'environnement conditionne la biologie des vecteurs (insectes, tiques), donc la diffusion de maladies à transmission vectorielle. Parlant des médicaments vétérinaires, l'oxytétracycline et l'Amitraz sont respectivement les molécules d'antibiotique et d'acaricide les plus utilisées par les éleveurs de la région du Poro. Le fort recours à l'oxytétracycline pourrait s'expliquer par son action curative efficace. En effet, l'injection de l'oxytétracycline à un animal malade lui permettrait d'obtenir rapidement la guérison. Ces résultats concordent à ceux de Camus et Morel (2000). Par ailleurs, le fort recours à l'Amitraz pourrait s'expliquer par son accessibilité et son coût relativement abordable. Ces résultats se rapprochent de ceux d'Achi et al. (2012) dans une étude menée dans le Nord de la Côte d'Ivoire et également de ceux d'Azokou et al. (2016) dans la même zone d'étude. Selon ces auteurs, l'Amitraz est le plus utilisé dans toutes les zones enquêtées. En effet, ce médicament vétérinaire a fait l'objet de vulgarisation par les agents de la Société pour le Développement de la Production Animale de la Côte d'Ivoire (Achi et al., 2012) et l'Agence Nationale d'Appui au développement Rural dénommée ANADER. En outre, malgré la forte utilisation de l'Amitraz, ce produit reste inefficace pour les éleveurs contre les tiques. Azokou et al. (2016) ont montré l'emploi de l'huile de vidange ou de moteur, l'utilisation de plantes médicinales et l'arrachage manuel dans la lutte contre les tiques. Codjia (2001) a relevé ces mêmes attitudes chez les éleveurs du Bénin où dans plusieurs campements, les éleveurs assurent eux-mêmes les injections avec tous les risques que cette pratique comporte (abcès, sous dosage et mauvaise dilution). Selon Morel (2000), la planification inappropriée du programme de contrôle des tiques peut être à l'origine de la résistance des tiques. Cette résistance des tiques à l'Amitraz a été montrée par de nombreux auteurs notamment Fernandez-Salas et al. (2012) et Lovis (2012) en Asie, en Afrique et en Australie.

$\mathrm{Au}$ niveau des doses d'antibiotique, $85,71 \%$ des éleveurs sédentaires injectent des doses faibles dans la lutte contre les maladies transmises par les tiques. Par contre, dans la lutte contre les tiques, la majorité des éleveurs ont recours à la sur-dilution des acaricides. Ce recours aux faibles doses des antibiotiques et l'économie du médicament peuvent être liés 
aux difficultés financières éprouvées par les éleveurs dans l'approvisionnement des médicaments. Par contre, le recours aux fortes doses des acaricides s'expliquerait par une probable résistance des tiques aux dilutions normales des acaricides et également par un détiquage inapproprié du bétail. Ces résultats se rapprochent de ceux obtenus par Bianchi et al. (2003) qui ont montré que le mode d'application manuelle des acaricides serait un facteur favorisant le développement de la résistance chez les tiques.

\section{Conclusion}

L'enquête menée dans la Région du Poro, a montré que les bovins et les ovins sont conduits sur le même pâturage dans tous les départements visités. Cette forme de conduite présente d'énormes conséquences notamment la persistance des tiques et par ricochet des agents pathogènes transmis par ces tiques au bétail. Les éleveurs de ces départements n'ont, pour la plupart, pas connaissance des maladies $\mathrm{du}$ bétail transmises par les tiques. Les dilutions inappropriées des médicaments ont été observées chez ces éleveurs interrogés. Une étude parasitologique dans la Région du Poro nous permettra d'inventorier les parasites transmis par les tiques afin de mieux proposer un plan de lutte contre ces affections qui minent sans cesse les élevages. L'identification de ces parasites transmis par les tiques permettra de mieux choisir les antibiotiques et les acaricides appropriés afin de réduire les dépenses effectuées par les éleveurs.

\section{CONFLIT D'INTERETS}

Les auteurs ne déclarent aucun conflit d'intérêt.

\section{CONTRIBUTIONS DES AUTEURS}

NY a fait les prélèvements, contribué à analyser les échantillons et rédiger la version initiale du manuscrit. YK et DS ont conçu cette étude et contribué à la discussion des résultats. ZFZB a révisé le manuscrit. SIT a contribué à analyser les échantillons.

\section{REMERCIEMENTS}

Les auteurs tiennent à remercier tous le personnel technique du Laboratoire Régional de Korhogo (LRK) et la Direction des Ressources Animales et Halieutiques de la Région du Poro pour avoir facilité les analyses et les prélèvements.

\section{REFERENCES}

Achi YL, Koné P, Stachurski F, Zinsstag J, Betschart B. 2012. Impact des tiques sur des bovins métissés dans le Nord de la Côte d'Ivoire. Bull. Anim. Hlth. Prod. Afr., 60(2): 109-118. URL: http://www.ajol.info/index.php/bahpa/artic le/view/81734.

Azokou A, Achi YL, Koné MW. 2016. Lutte contre les tiques du bétail en Côte d'Ivoire par des méthodes traditionnelles. Livestock Research for Rural Development, 28(52). URL:http://www.lrrd.org/lrrd28/4/azok280 52.htm.

Banchi MW, Barré N, Messad S. 2003. Factors related to cattle infestation level and resistance to acaricides in Boophilus microplus tick populations in New Caledonia. Veterinary Parasitology, 112(1-2): 75-89. DOI: http://dx.doi.org/10.1016/S03044017(02)00415-6.

Camus E, Morel PC. 2000. Précis de parasitologie vétérinaire tropicale. Universités francophones, Paris, Tec et Doc ; 645-656.

Codjia V. 2001. Rapport de consultation. Contribution à l'analyse cout/bénéfice des scénarios de lutte contre les mouches tsétsé et les trypanosomoses en Afrique de l'Ouest. Données de la zone d'étude au Bénin, p.185. URL: wwwnaweb.iaea.org/nafa/aph/public/annex3tsetse. 
Diallo Y. 2007. «Les Peuls, les Sénoufo et l'État au nord de la Côte d'Ivoire. Problèmes fonciers et gestion du pastoralisme. », Bulletin de l'APAD, 10 | 1995.

URL :

http://apad.revues.org/1131.

Djakaridja B, Yao KP, Gragnon BG, AcapoviYao G, Mavoungou JL. 2014. Situation épidémiologique des hémoparasites des bovins dans deux zones d'élevage de la Côte d'Ivoire: cas des anciennes régions des savanes et de la vallée Bandama. Revue Méd. Vét., 165: 297-303. URL: www.revmedvet.com/2014/RMV165_297 303 .

Farougou S, Tassou AW, Tchabode DM, Kpodekon M, Boko C, Youssao AKI. 2007. Tiques et hémoparasites du bétail dans le nord-Bénin. Revue Méd. Vét., 158: 463-467. URL: www.revmedvet.com/2007/RMV158_463 467.pdf.

Fernández-Salas A, Rodríguez-Vivas RI, Alonso-Díaz MA. 2012. Resistance of Rhipicephalus microplus to Amitraz and Cypermethrin in Tropical Cattle Farms in Veracruz, Mexico. Journal of Parasitology, 98(5): 1010-1014. DOI: 10.1645/GE-3074.1.

Lancelot R, Zundel E, Ducrot C. 2011. Spécificités de la santé animale en régions chaudes : le cas des maladies infectieuses majeures en Afrique. INRA Prod. Anim., 24(1): 65-76. URL : http://www6.inra.fr/productionsanimales/2011-Volume-24/Numero-12011/Specificites-de-la-sante-animale-enregions-chaudes-le-cas-des-maladiesinfectieuses-majeures

Lovis L. 2012. Evaluation of acaricide resistance in the cattle tick, Rhipicephalus (Boophilus) microplus, using a new in vitro test and molecular Tools. PhD thesis,
Neuchâtel University, Neuchâtel, p.204. URL: urn:nbn:ch:rero-004-111114

Minjauw B, McLeod A. 2003. Tick-borne diseases and poverty. The impact of ticks and tick-borne diseases on the livelihood and marginal livestock owners in India and Eastern and Southern Africa. Research report, DFID Animal Health Programme, Centre of Tropical Veterinary Medicine, University of Edinburgh, U K: 59-60. URL:

https://www.cabdirect.org/cabdirect/abstra ct/20063155090.

Morel PC. 2000. Maladies à tiques du bétail en Afrique. In Précis de Parasitologie Vétérinaire Tropicale, Chartier C, Itard J, Morel PC, Troncy PM (Eds). Editions Médicales internationales, Cachan, Editions Tec et Doc : Paris ; 452-761.

Rebaud A. 2006. Eléments d'épidémiologie de la babesiose bovine à Babesia divergens dans une clientèle des monts du Lyonnais. Thèse de Doctorat, Université Claude Bernard - Lyon 1, Lyon, p.95. URL : www2.vetagrosup.fr/bib/fondoc/th_sout/dl.php?file=2006 lyon003.pdf

Tanguy Le G. 2004. Le développement agricole et pastorale du nord de la Côte d'Ivoire: Problème de coexistence, les cahiers d'outre-mer : 226-227. DOI: $10.4000 /$ com.563.

Yabile RK. 2011. Promotion de l'agriculture vivrière et croissance économique de la Côte d'Ivoire : quelle relation? Agronomie Africaine 23(3): 259-272. URL :http://www.ajol.info/index.php/aga/ article/view/77823 